Article

\title{
Bandwidth Optimization Design of a Multi Degree of Freedom MEMS Gyroscope
}

\author{
Chaowei Si ${ }^{1,2}$, Guowei Han ${ }^{1}$, Jin Ning ${ }^{1, *}$ and Fuhua Yang ${ }^{1, *}$ \\ 1 Institute of Semiconductors, Chinese Academy of Science, Beijing 100083, China; \\ E-Mails: schw@semi.ac.cn (C.S.); hangw1984@semi.ac.cn (G.H.) \\ 2 Department of Electronic Engineering, Tsinghua University, Beijing 100084, China \\ * Authors to whom correspondence should be addressed; E-Mails: ningjin@ semi.ac.cn (J.N.); \\ fhyang@semi.ac.cn (F.Y.); Tel.: +86-10-8230-4492 (J.N.); +86-10-8230-5100 (F.Y.).
}

Received: 22 June 2013; in revised form: 3 August 2013 / Accepted: 9 August 2013 /

Published: 14 August 2013

\begin{abstract}
A new robust multi-degree of freedom (multi-DOF) MEMS gyroscope is presented in this paper. The designed gyroscope has its bandwidth and amplification factor of the sense mode adjusted more easily than the previous reported multi-DOF MEMS gyroscopes. Besides, a novel spring system with very small coupling stiffness is proposed, which helps achieve a narrow bandwidth and a high amplification factor for a 2-DOF vibration system. A multi-DOF gyroscope with the proposed weak spring system is designed, and simulations indicate that when the operating frequency is set at $12.59 \mathrm{kHz}$, the flat frequency response region of the sense mode can be designed as narrow as $80 \mathrm{~Hz}$, and the amplification factor of the sense mode at the operating frequency is up to 91 , which not only protects the amplification factor from instability against process and temperature variations, but also sacrifices less performance. An experiment is also carried out to demonstrate the validity of the design. The multi-DOF gyroscope with the proposed weak coupling spring system is capable of achieving a good tradeoff between robustness and the performance.
\end{abstract}

Keywords: multi-DOF MEMS gyroscope; robustness; narrow bandwidth; large amplification factor 


\section{Introduction}

Most studies on MEMS gyroscopes are focused on their performance, and common methods to improve the performance include improving quality factor enhancement [1], quadrature error cancellation [2], and mode matching [3], based on which the bias drift has already been driven below 0.1 degrees/h [4,5]. On the other side, the robustness has also been considered, and multi-DOF MEMS gyroscopes are proved to display good robustness and long-term stability, as they are insensitive to structural and environmental parameter variations [6-8].

A series of tests on 3-DOF MEMS gyroscopes show that the amplification factor is insensitive to temperature variation, power supply jitter and damping change [9]. A more robust multi-DOF gyroscope with 2-DOF sense mode and 2-DOF drive mode is further reported [10], but the improvement of the robustness affects the sensitivity.

For the 2-DOF vibration system consisted of two spring mass systems in series described in [6-11], the amplification factor of the 2-DOF vibration mode is inversely proportional to the square of the bandwidth [11], but unfortunately, the bandwidth is hard to narrow enough to get ideal amplification factor, because the bandwidth is determined by the spring stiffness ratio and the mass ratio, and those ratios are within limits due to process capacities.

The paper adopts another 2-DOF vibration system consisted of two spring mass systems connected by a coupling spring, from the equivalent mechanical model, it is found that the increase of the amplification factor relies on the decrease of the coupling spring stiffness. Furthermore, a novel spring system of very small coupling spring stiffness is proposed. Simulations of a reasonably designed gyroscope with the proposed spring system indicate that the amplification factor is up to 91 when the peak to peak bandwidth is narrowed to $240 \mathrm{~Hz}$.

\section{Work Principle and Design}

\subsection{Analysis of 2-DOF Vibration Systems}

Generally, in the fabrication of a MEMS gyroscope, resonant frequency drift caused by the process variations could be up to tens of Hertz [7]. Meanwhile, the resonant frequency separation of the drive mode and the sense mode varies with the operating temperature, both of which influence the performance. The performance loss due to the fabrication errors and the temperature variations can be prevented if the frequency response in the sense mode is flat in a specific range [9], and for all reported multi-DOF gyroscopes [6-11], the bandwidth meets the demand, but a large bandwidth results in a small amplification factor, in other words, the sensitivity of the gyroscope is decreased [11].

The 2-DOF vibration systems mentioned in [6,9] consist of two spring mass systems in series, and the way to enlarge the amplification factor is to make it operate in a low central frequency and decrease the mass ratio and the spring stiffness ratio. To realize a flat region about $200 \mathrm{~Hz}$, the drive mode resonant frequency is designed to be $752 \mathrm{~Hz}$, with a mass ratio of 0.0624 and a spring stiffness ratio of 0.0429 in [9]. Moreover, the mass ratio is set to 0.05 when the central frequency is about $2 \mathrm{kHz}$ in [6]. It is difficult to narrow the bandwidth further for larger mass ratio and spring stiffness ratio requires smaller masses and longer folded beams, which are limited by the current fabrication capabilities. 
It is less complicated to adjust the bandwidth of a 2-DOF vibration system consisting of two spring mass systems connected by a coupling spring, whose equivalent mechanical model is shown in Figure 1.

Figure 1. The mechanical model of a 2-DOF vibration system.

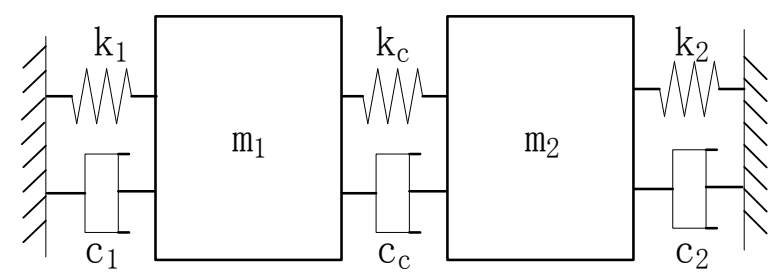

One of the spring mass systems consists of a support spring $k_{1}$, a mass $m_{1}$ and the damping factor is $c_{1}$, the other is composed of a support spring $k_{2}$, a mass $m_{2}$ and the damping factor is $c_{2}$. The two spring mass systems are connected by a coupling spring $k_{c}$ with an extra damping factor $c_{c}$ introduced. The resonant frequency of the first spring mass system added with $k_{c}$ is $\omega_{s 1}$, the resonant frequency the latter added with $k_{c}$ is $\omega_{s 2}$, that means $\omega_{s 1}^{2}=\left(k_{1}+k_{c}\right) / m_{1}, \omega_{s 2}{ }^{2}=\left(k_{2}+k_{\mathrm{c}}\right) / m_{2}$. The spring stiffness ratio of $k_{c}$ to $k_{1}$ is $r_{c 1}$, the spring stiffness ratio of $k_{c}$ to $k_{2}$ is $r_{c 2}$, the central frequency is $\omega_{0}$, and the two peak frequencies of the 2-DOF system are $\omega_{1}$ and $\omega_{2}$, which are revealed in Equations (1) and (2):

$$
\begin{aligned}
& \omega_{1}{ }^{2}=\frac{\omega_{s 2}{ }^{2}}{2}+\frac{\omega_{s 1}{ }^{2}}{2}+\sqrt{\left(\frac{\omega_{s 2}{ }^{2}}{2}-\frac{\omega_{s 1}{ }^{2}}{2}\right)^{2}+\frac{r_{c 1} r_{c 2}}{\left(1+r_{c 1}\right)\left(1+r_{c 2}\right)} \omega_{s 1}{ }^{2} \omega_{s 2}{ }^{2}} \\
& \omega_{2}{ }^{2}=\frac{\omega_{s 2}{ }^{2}}{2}+\frac{\omega_{s 1}{ }^{2}}{2}-\sqrt{\left(\frac{\omega_{s 2}{ }^{2}}{2}-\frac{\omega_{s 1}{ }^{2}}{2}\right)^{2}+\frac{r_{c 1} r_{c 2}}{\left(1+r_{c 1}\right)\left(1+r_{c 2}\right)} \omega_{s 1}{ }^{2} \omega_{s 2}{ }^{2}}
\end{aligned}
$$

The peak to peak bandwidth $\omega_{1}-\omega_{2}$ is defined as $B W_{p p}$. Obviously, $B W_{p p}$ get a minimum when $\omega_{s 1}=\omega_{s 2}=\omega_{s}$. Assuming $p^{2}=r_{c 1} r_{c 2} /\left(\left(1+r_{c 1}\right)\left(1+r_{c 2}\right)\right), B W_{p p}$ is derived in Equation (3):

$$
B W_{p p}=\omega_{2}-\omega_{1}=(\sqrt{1+p}-\sqrt{1-p}) \omega_{s} \approx p \omega_{s}
$$

By truncating appropriately the Taylor series expansion for $\sqrt{1+p}$ and $\sqrt{1-p}, B W_{p p}$ is acquired in Equation (4):

$$
B W_{p p} \approx p \omega_{s}
$$

and the central frequency $\omega_{0}$ is derived in Equation (5):

$$
\omega_{0}=\frac{\omega_{2}+\omega_{1}}{2}=\frac{(\sqrt{1+p}+\sqrt{1-p})}{2} \omega_{s}
$$

Taylor series expansions are used to simplify the above equation, and a simplified equation of $\omega_{0}$ is given in Equation (6).

$$
\omega_{0} \approx \omega_{s}
$$

From Equation (4), $B W_{p p}$ is proportional to $p$ when $\omega_{s}$ is given, so the bandwidth optimization is simplified as there is only one parameter to adjust.

In a MEMS gyroscope, a spring is often made up of a number of folded beams in series, and the stiffness of the spring is inversely proportional to the total effective length. According to the above 
analyses, narrow bandwidth requires a coupling spring of very small stiffness, and if $k_{c}$ is made up of traditional folded beams, it should have a length tens of the support spring to achieve a very narrow bandwidth. As revealed in [9], the large spring stiffness ratio is achieved by increasing the number of folded beams in series, whereby not only is a large area occupied, but the resonant frequency of the coupling spring also decreases, which may disturb the operation of the gyroscope, so a new kind of spring structure with a very small coupling stiffness is necessary.

\subsection{Design of a Weak Coupling Spring System}

The designed weak coupling spring system consists of three beams: two parallel beams a and $b$ are fixed on a vertical beam c, both ends of c are fixed, as shown in Figure 2. Beams a and b are used as support springs, the beam c serves as the coupling spring.

Figure 2. The weak coupling spring system. (a) The weak coupling spring system structure; (b) Deformation of the spring system when a force is applied.

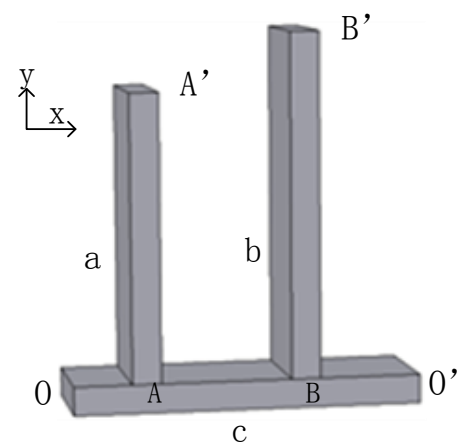

(a)

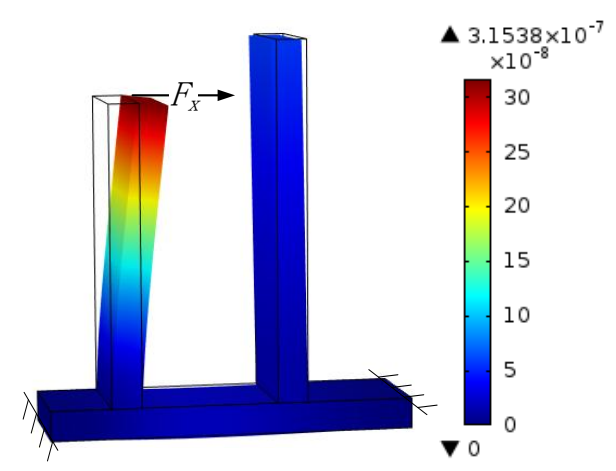

(b)

To describe the mechanism of the spring system conveniently, two ends of the beam c are set as $\mathrm{O}$ and $\mathrm{O}^{\prime}$, the joint of beams a and $\mathrm{c}$ is $\mathrm{A}$, the joint of beams b and c is $\mathrm{B}$, the free end of the beam a is $\mathrm{A}^{\prime}$, the free end of the beam $b$ is $B^{\prime}$, as shown in Figure $2 a$.

The support spring stiffness is considered first. When a force $F_{x}$ is applied on one support spring such as the point of $\mathrm{A}^{\prime}$ of the beam a along the $\mathrm{x}$ axis, there is an angle between the line $\mathrm{OA}^{\prime}$ and the direction of the force $F_{x}$, hence the united corner beam OA and a is rotated around $\mathrm{O}$ under the action of the torque $\overrightarrow{F_{x}} \times \overrightarrow{O A^{\prime}}$. Moreover, there is another torque $\overrightarrow{F_{x}} \times \overrightarrow{O^{\prime} A^{\prime}}$ that makes the united corner beam $\mathrm{O}^{\prime} \mathrm{A}$ rotate around $\mathrm{O}^{\prime}$. Under the action of the resultant moment, the beam c is bent, as well as the beam a. The net motion of $\mathrm{A}^{\prime}$ is dependent on the combined deformation of the bended beam $\mathrm{c}$ and the bended beam a, and the support spring stiffness of the beam a is the ratio of the force $F_{x}$ to the net motion of $\mathrm{A}^{\prime}$.

Considering the traditional coupling spring form that two support springs are connected by a coupling spring, as shown in Figure 1, if a force is applied on the joint of $k_{c}$ and $k_{l}$ along the $\mathrm{x}$ axis, $k_{c}$ is compressed much more than $k_{2}$ due to its small stiffness, and the joint of $k_{c}$ and $k_{2}$ moves much less than the former joint. Considering the weak coupling spring system in Figure 2, when the beam $\mathrm{c}$ is bent, the beam $\mathrm{b}$ rotates around $\mathrm{O}^{\prime}$ along with the motion of $\mathrm{B}$, and the net motion of $\mathrm{B}^{\prime}$ is very small. The deformation state of the weak coupling spring system under the action of a force $F_{x}$ along the 
$\mathrm{x}$ axis on the point $\mathrm{A}^{\prime}$ is simulated, as shown in Figure $2 \mathrm{~b}$. As revealed, the motion of $\mathrm{A}^{\prime}$ is far larger than the motion of $\mathrm{B}^{\prime}$, which indicates that the coupling spring stiffness is far smaller than the support spring stiffness.

On the basis of the above analysis, it can be inferred that the shorter the segment $\mathrm{BO}^{\prime}$ is, the less the motion of $\mathrm{B}^{\prime}$ is, and the smaller the coupling spring stiffness is. On the other hand, the decrease of the segment $\mathrm{OA}$ leads to the decrease of the resultant moment of $\overrightarrow{F_{x}} \times \overrightarrow{O A^{\prime}}$ and $\overrightarrow{F_{x}} \times \overrightarrow{O^{\prime} A^{\prime}}$, so the beam c is bent less, and the coupling spring stiffness is reduced. The resultant moment reaches max when $\mathrm{A}$ is in the middle of beam c.

\subsection{Design of a MEMS Gyroscope with a Weak Coupling Spring System}

The designed gyroscope is a single DOF vibration system in the drive direction, as well as a 2-DOF vibration system in the sense direction. The frame structure is used for minimizing the quadrature error, as shown in Figure 3. The drive mass $m_{d}$ is fixed on the active sense mass $m_{1}$ by the spring $k_{\mathrm{d}}$, the passive sense mass $m_{2}$ is out of $m_{1}$ and connected to $m_{1}$ with a weak coupling spring system $\mathrm{K}_{\text {couple, of }}$ which the equivalent support spring stiffness is $k_{e q 1}$ and $k_{e q 2}$, equal coupling spring stiffness is $k_{e q c}$. The mechanical model is established to describe to the vibration of masses, as shown in Figure $3 b$.

Figure 3. The MEMS gyroscope with a weak coupling spring system. (a) Schematic of the designed gyroscope; (b) Mechanical mode of the designed gyroscope.

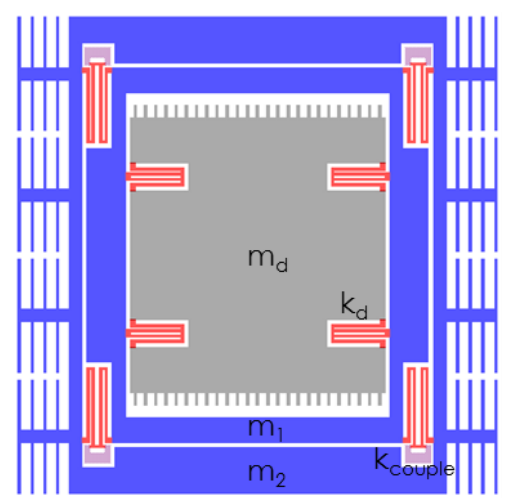

(a)

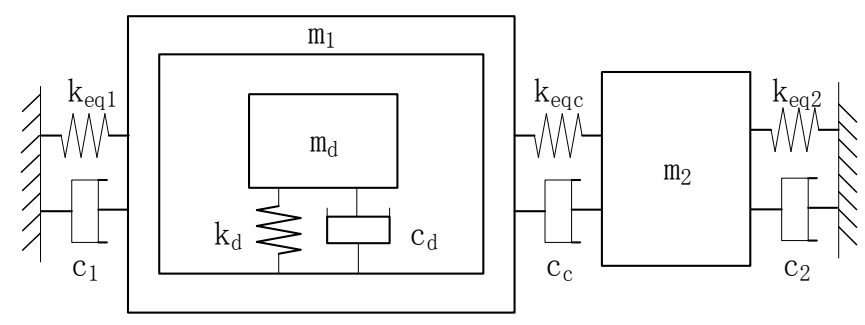

(b)

The drive mode is shown in Figure 4a, and the two sense modes are shown in Figure 4b,c. At the in-phase mode frequency, the masses move in phase, while at the anti-phase mode frequency, the masses move oppositely. The central frequency of the sense mode is in the middle of the in-phase mode frequency and the anti-phase mode frequency, near which the frequency response of $m_{2}$ is flat. The gyroscope operates at the drive mode resonant frequency, which is designed at the central frequency, so the amplification factor changes little when the operation frequency drifts in a certain range [6].

Assuming the displacement of $m_{d}$ is $x_{d}$, and the applied force on it is $F_{d}(t)$, the displacement Equation of $m_{d}$ along the drive direction is:

$$
m_{d} x_{d}^{\prime \prime}+c_{d} x_{d}^{\prime}+k_{d} x_{d}==F_{d}(t)
$$


Figure 4. Vibration modes of the designed MEMS gyroscope. (a) Drive mode; (b) In-phase sense mode; (c) Anti-phase sense mode.

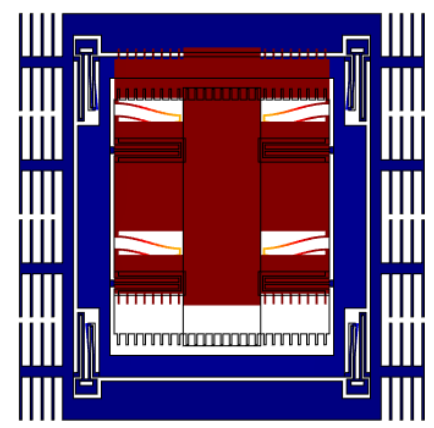

(a)

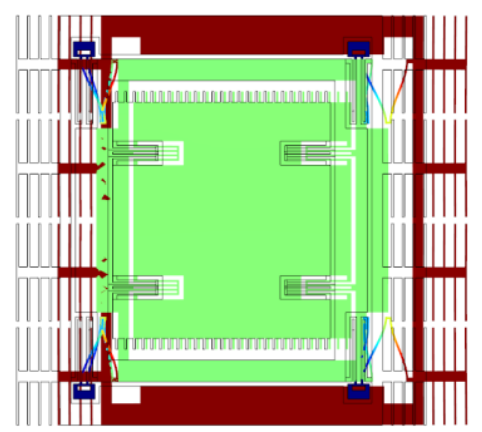

(b)

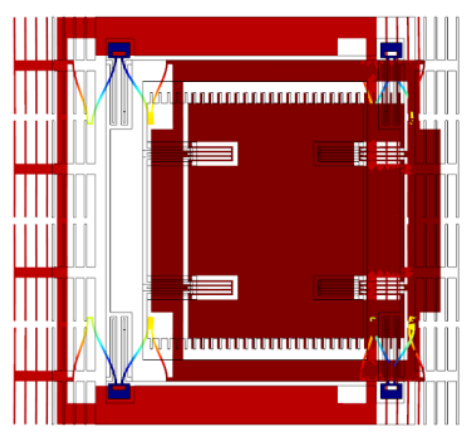

(c)

The displacement equations of $m_{1}$ and $m_{2}$ along the sense direction are:

$$
\begin{gathered}
\left(m_{d}+m_{1}\right) x_{1}^{\prime \prime}+\left(c_{1}+c_{c}\right) x_{1}^{\prime}-c_{c} x_{2}^{\prime}+k_{e q 1} x_{1}=k_{e q c}\left(x_{2}-x_{1}\right)+F_{c}(t) \\
m_{2} x_{2}^{\prime \prime}+\left(c_{2}+c_{c}\right) x_{2}^{\prime}-c_{c} x_{1}^{\prime}+k_{e q 2} x_{2}=k_{e q c}\left(x_{1}-x_{2}\right)
\end{gathered}
$$

wherein, $x_{1}$ represents the displacement of $m_{1}, x_{2}$ represents the displacement of $m_{2}, F_{c}(t)$ is the Coriolis force sensed by $m_{d}$, and can be expressed as $F_{c}(t)=2 m_{d} \omega_{d} x_{l}^{\prime}(t), \omega_{d}$ is the resonant frequency of $m_{d}$.

Based on the above analysis, the bandwidth minimum is only determined by the coupling spring stiffness in case of $\left(k_{e q 1}+k_{e q c}\right) /\left(m_{d}+m_{1}\right)=\left(k_{e q 2}+k_{e q c}\right) / m_{2}=\omega_{s}^{2}$. The ratio of $k_{e q c}$ to $k_{e q 1}$ is defined as $r_{c 1}$, the ratio of $k_{e q c}$ to $k_{e q 2}$ is defined as $r_{c 2}$. Equations (8) and (9) are rewritten in the frequency domain, as shown below:

$$
\begin{gathered}
s^{2}\left(m_{1}+m_{d}\right) X_{1}(s)+s\left(c_{1}+c_{c}\right) X_{1}(s)-s c_{c} X_{2}(s)+k_{e q 1} X_{1}(s)=k_{e q c}\left(X_{2}(s)-X_{1}(s)\right)+F_{c}(s) \\
s^{2} m_{2} X_{2}(s)+s\left(c_{2}+c_{c}\right) X_{2}(s)-s c_{c} X_{1}(s)+k_{e q 2} X_{2}(s)=k_{e q c}\left(X_{1}(s)-X_{2}(s)\right)
\end{gathered}
$$

From Equation (11), the relation between $X_{1}(\mathrm{~s})$ and $X_{2}(s)$ is derived and shown in Equation (12), and the transition function $H_{2}(s)$ of the displacement $X_{2}(s)$ against the Coriolis force $F_{c}(s)$ is expressed in Equation (13):

$$
\begin{gathered}
X_{1}(s)=\frac{s^{2} m_{2}+s\left(c_{2}+c_{c}\right)+k_{e q 2}+k_{e q c}}{k_{e q c}+s c_{c}} X_{2}(s) \\
H_{2}(s)=\frac{k_{e q c}+s c_{c}}{m_{2}\left(m_{1}+m_{d}\right)} \frac{1}{\left(s^{2}+\frac{c_{1}+c_{c}}{m_{1}+m_{d}} s+\omega_{s}^{2}\right)\left(s^{2}+\frac{c_{2}+c_{c}}{m_{2}} s+\omega_{s}^{2}\right)-\frac{\left(k_{e q c}+s c_{c}\right)^{2}}{m_{2}\left(m_{1}+m_{d}\right)}}
\end{gathered}
$$

When $s=j \omega_{0} \approx j \omega_{s}$, the gain of $x_{2}$ is:

$$
H_{2}\left(j \omega_{s}\right)=\frac{k_{e q c}+j \omega_{s} c_{c}}{-\left(c_{1}+c_{c}\right)\left(c_{2}+c_{c}\right) \omega_{s}^{2}-\left(k_{e q c}+j \omega_{s} c_{c}\right)^{2}}
$$

Equation (14) reveals that if all the damping factors are zero, $\left|H_{2}\left(j \omega_{s}\right)\right|$ is inversely proportional to $k_{e q c}$, and if damping factors exits, $\left|H_{2}\left(j \omega_{s}\right)\right|$ increases also along with the decrease of $k_{\text {eqc }}$. According to 
the above analysis, it can be inferred that the designed gyroscope with the weak spring system has optimized the amplification factor of the sense mode.

\section{Results and Discussion}

The layout and a fabricated gyroscope are shown in Figure 5, wherein interdigitated comb-drivers are used to drive the gyroscope, groups of parallel-plate capacitors serves as the sense structure, the quadrature adjustment electrodes are on sides of the sense electrodes, and extra test electrodes are placed to sense the motion of $m_{1}$.

Figure 5. Layout of the designed gyroscope. (a) Layout and partial photo of the designed gyroscope; (b) Photo of the designed weak coupling spring system.

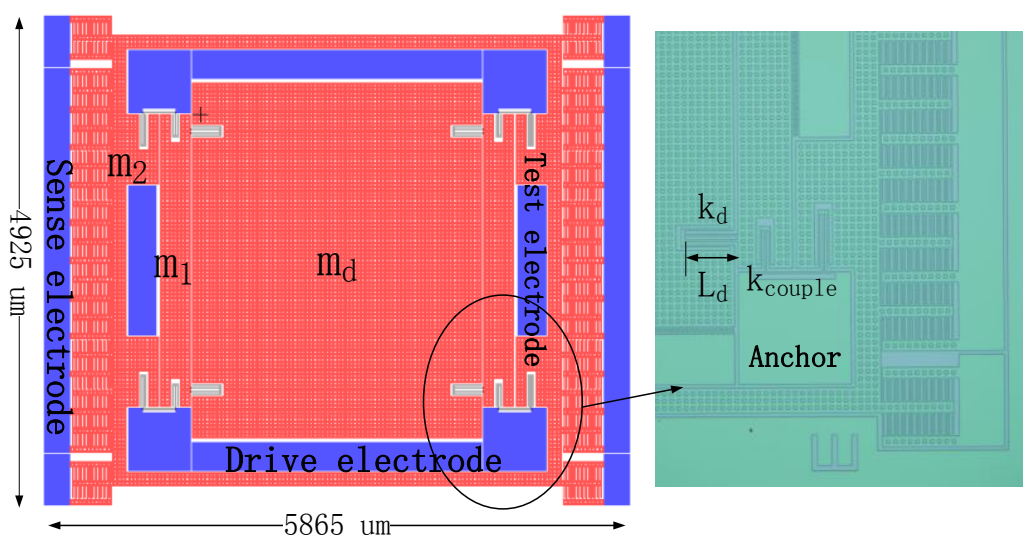

(a)

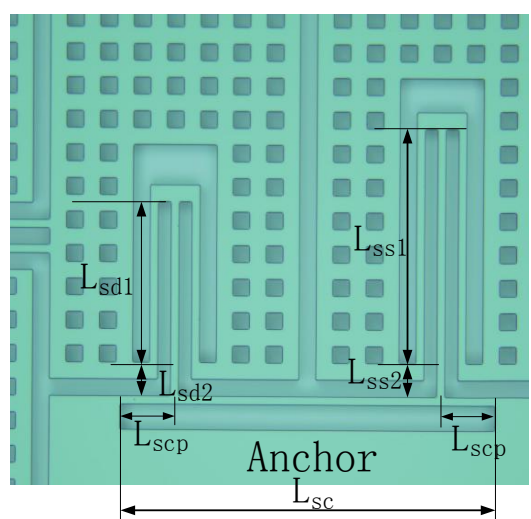

(b)

Each support spring of $m_{d}$ consists of four folded beams to achieve good linearity, while each support spring of $m_{1}$ or $m_{2}$ consists of three folded beams, they are joined on a coupling spring as shown in Figure 5b. Since the coupling spring stiffness is dependent on the distances between anchors and joints, the springs of three folded beams are capable of having joints nearer to the anchors with less fixed area.

The proposed gyroscope is fabricated on a n-type (100) silicon on insulator (SOI) wafer, with a thickness of $20 \mu \mathrm{m}$, the designed width of all beams are $10 \mu \mathrm{m}$, and all holes on the structure are $20 \times 20 \mu \mathrm{m}^{2}$. The other parameters are shown in Table 1 .

Table 1. Key parameters of the designed gyroscope.

\begin{tabular}{cccc}
\hline Designed Parameters & Value & Designed Parameters & Value \\
\hline $\mathrm{L}_{\mathrm{d}}(\mu \mathrm{m})$ & 291.5 & $\mathrm{~m}_{\mathrm{d}}(\mathrm{mg})$ & 0.232 \\
$\mathrm{~L}_{\mathrm{ss} 1}(\mu \mathrm{m})$ & 279 & $\mathrm{~m}_{1}(\mathrm{mg})$ & 0.122 \\
$\mathrm{~L}_{\mathrm{ss} 2}(\mu \mathrm{m})$ & 40 & $\mathrm{~m}_{2}(\mathrm{mg})$ & 0.198 \\
$\mathrm{~L}_{\mathrm{sd} 1}(\mu \mathrm{m})$ & 192 & $\mathrm{~K}_{\mathrm{d}}(\mathrm{KN} / \mathrm{m})$ & 1.49 \\
$\mathrm{~L}_{\mathrm{sd} 2}(\mu \mathrm{m})$ & 40 & $\mathrm{~K}_{\mathrm{eq} 1}(\mathrm{KN} / \mathrm{m})$ & 1.26 \\
$\mathrm{~L}_{\mathrm{sc}}(\mu \mathrm{m})$ & 340 & $\mathrm{~K}_{\mathrm{eq} 2}(\mathrm{KN} / \mathrm{m})$ & 2.27 \\
$\mathrm{~L}_{\mathrm{scp}}(\mu \mathrm{m})$ & 10 & $\mathrm{~K}_{\mathrm{eqc}}(\mathrm{KN} / \mathrm{m})$ & 0.056 \\
\hline
\end{tabular}


The gyroscope structure was fabricated using ICP etching, before which Al was ion beam sputtering deposited as etching mask. After the $\mathrm{Al}$ was corroded, the movable structure was released by etching sacrificial silicon dioxide in $49 \%$ aqueous $\mathrm{HF}$ solutions at $60{ }^{\circ} \mathrm{C}$. The release process takes about $280 \mathrm{~s}$. The central frequency amplification factor of the 2-DOF vibration system shown in Figure 1 is determined by damping factors as well as the coupling spring stiffness. The effect of damping factors is considered here first. Since anchor loss is the main energy loss mechanism when the gyroscope operates in high vacuum, the impact of $c_{c}$ can be ignored [12]. In order to make the simulation simple, the quality factors of each spring mass system are assumed to be equal, and their value is Q. The frequency response of the passive mass $m_{2}$ against damping factors is simulated, as shown in Figure 6a, which indicates that when $\mathrm{Q}$ changes above 1,000, the central frequency amplification factor is invariant. Quality factors of most MEMS gyroscopes are over 1,000, so the designed gyroscope is insensitive to damping factor variations, and it is reasonable to use 1,000 as the value of $\mathrm{Q}$ in the next simulations.

Figure 6. Simulated frequency response of the designed MEMS gyroscope. (a) Frequency response of $m_{2}$ against damping factors at room temperature; (b) The sense mode frequency response against temperature variations; (c) The drive mode frequency response of against temperature variations.

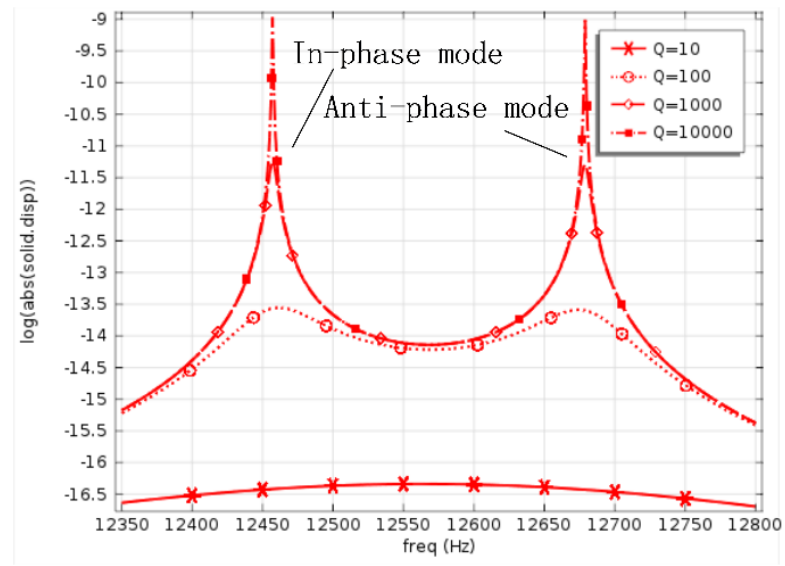

(a)

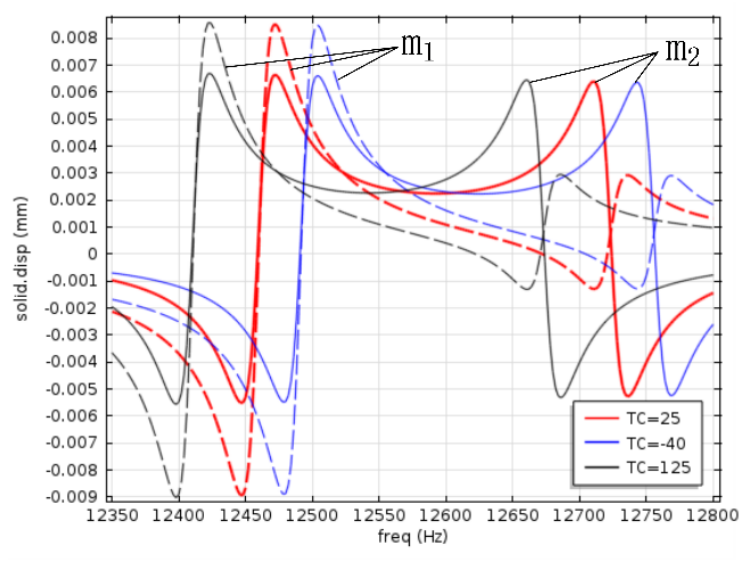

(b)

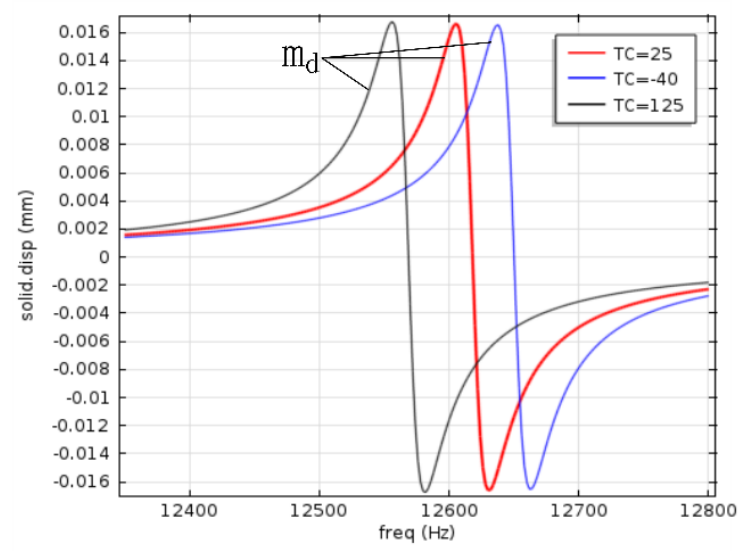

(c) 
In Figure 6b, the solid lines are displacement-frequency response curves of $m_{2}$, dotted lines are displacement-frequency response curves of $m_{1}$ in the sense direction. At room temperature, the central frequency is $12.59 \mathrm{kHz}$, the frequencies of two modes are $12.47 \mathrm{kHz}$ and $12.71 \mathrm{kHz}$, the peak to peak bandwidth is about $240 \mathrm{~Hz}$, and the amplification factor at the central frequency is 91, which reveals a good tradeoff between the bandwidth and the amplification factor.

Both frequency responses of the drive mode and the sense mode are sensitive to the dimensional variations due to fabrication tolerances, and the resonant frequency separation induced by stable process is usually less than tens of Hertz [7]. Simulation results in Figure 6b reveal that the amplification factor varies up to $10 \%$ when the operation frequency drifts from $12.55 \mathrm{kHz}$ to $12.63 \mathrm{kHz}$ at room temperature, having a flat range as wide as $80 \mathrm{~Hz}$, so even if there is a frequency separation caused by process variations, the operation frequency is still located in the flat region, which indicates that the amplification factor of the designed 2-DOF gyroscope is insensitive to fabrication errors.

Young's modulus $E$ and Poisson's ratio $v$ of silicon are dependent on crystal directions as well as the temperature, and the temperature coefficient of $E$ for the [110] direction is $-131 \times 10^{-6}{ }^{\circ} \mathrm{C}$ at room temperature [13], so the frequency response varies with temperature. The displacement-frequency response of $m_{\mathrm{d}}$ against temperature is simulated and shown in Figure 6c, and that of $m_{2}$ and $m_{1}$ is shown in Figure 6(b), wherein red lines represent the frequency response at $25^{\circ} \mathrm{C}$, gray lines represent the frequency response at $125^{\circ} \mathrm{C}$, and blue lines represent the frequency response at $-40{ }^{\circ} \mathrm{C}$. It can be seen that the resonant frequency of $m_{\mathrm{d}}$ varies $3 \mathrm{~Hz}$ from $-40{ }^{\circ} \mathrm{C}$ to $25{ }^{\circ} \mathrm{C}$, and $5 \mathrm{~Hz}$ from $25{ }^{\circ} \mathrm{C}$ to $125^{\circ} \mathrm{C}$, while the central frequency of $m_{2}$ varies $6 \mathrm{~Hz}$ from $-40{ }^{\circ} \mathrm{C}$ to $25^{\circ} \mathrm{C}$, and $10 \mathrm{~Hz}$ from $25^{\circ} \mathrm{C}$ to $125{ }^{\circ} \mathrm{C}$, revealing that the resonant frequency of $m_{\mathrm{d}}$ is always in the flat region of the frequency response of $m_{2}$ within the whole temperature range. Therefore, the designed 2-DOF gyroscope is insensitive to temperature variations.

The frequency response of the 2-DOF vibration system in the designed MEMS gyroscope was tested with a Scanning Laser Doppler Vibrometer. The gyroscope was placed on a PZT in a sealed tank with the air pressure $6.7 \mathrm{~Pa}$ at about $22{ }^{\circ} \mathrm{C}$ in test, an AC exciting force is applied on the substrate side of the gyroscope along the sense direction, the force is provided by a PZT exited by a group of periodical chirp electric signals, and the vibration signal of microstructure was acquired by the Scanning Laser Doppler Vibrometer.

The amplitude-frequency and phase- frequency response of different masses is shown in Figure 7, gray lines represent the frequency response of the mass $m_{2}$, and red lines represent the frequency response of the mass $m_{2}$. Due to fabrication errors, the measured central frequency is about $200 \mathrm{~Hz}$ below the simulation, and the tested peak to peak bandwidth is about $284 \mathrm{~Hz}$, both are still in agreement with the simulation result capable of meeting the needs of practical application. The vibration magnitude at the central frequency $12.302 \mathrm{kHz}$ is $0.384 \mathrm{~nm}$, and the magnitude far from resonant frequencies is about $9 \mathrm{pm}$, from which the amplification factor at the central frequency is about 42.7 , not as good as the simulation value, which is due to different stimulation ways on masses between in test and in simulation, but the result is still encouraging. The flat range is $118 \mathrm{~Hz}$ when the magnitude varies $10 \%$ compared to the magnitude at the central frequency.

Compared to the former multi-DOF gyroscopes described in [6-11], the designed MEMS gyroscope with the weak coupling spring system is capable of operating at higher frequency, having narrower bandwidth as well as larger amplification factor, and shows the potential to make a better tradeoff 
between the amplification factor and the bandwidth. Besides, the size design is more flexible, without extreme sizes to realize narrow bandwidth.

Figure 7. The tested sense mode frequency response of the fabricated MEMS gyroscope.

Signal: FFT - Vib Displacement - Mag. \& Phase
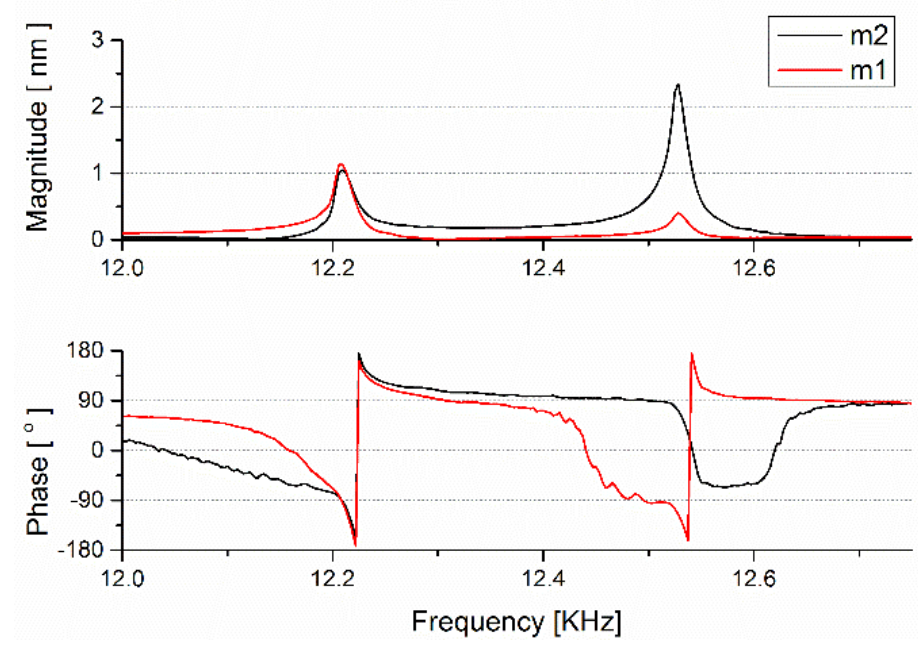

\section{Conclusions}

The performance of a MEMS gyroscope is influenced a lot by the mechanical impedance, and due to the decrease of the amplification factor, the mechanical impedance of gyroscopes with 2-DOF sense mode is increased compared with that of gyroscopes with single DOF sense mode, so the sensitivity and the bias stability of a multi-DOF MEMS gyroscope is not that good in theory, but the robustness is attractive. Still there are ways to improve the performance of multi-DOF MEMS gyroscope, like improving the quality factor of the drive mode, or enlarging the amplification factor of the sense mode.

The 2-DOF vibration model used in this paper has parameters adjusted more conveniently to make a tradeoff between the bandwidth and the amplification factor. Besides, a weak coupling spring system of tiny stiffness is proposed to achieve a narrow bandwidth, and the designed gyroscope with the weak coupling spring system has an amplification factor of 91 at with the central frequency, proving good robustness while no sacrificing sensitivity.

\section{Acknowledgments}

The authors gratefully acknowledge Chinese National Science Foundation's financial support (Contract No. 61274001, No. 61006073, and No. 61234007) and National Hi-Tech Research and Development Program (Contract No. 2006AA04Z339).

\section{Conflicts of Interest}

The authors declare no conflict of interest. 


\section{References}

1. Sharma, A.; Zaman, F.M.; Amini, B.V.; Ayazi, F. A high-Q in-plane SOI tuning fork gyroscope. Proc. IEEE Sens. 2004, 1-3, 467-470.

2. Tatar, E.; Alper, S.E.; Akin, T. Effect of Quadrature Error on the Performance of a Fully-Decoupled MEMS Gyroscope. In Proceedings of 2011 IEEE 24th International Conference on Micro Electro Mechanical Systems (MEMS), Cancun, Mexico, 23-27 January 2011; pp. 569-572.

3. Sharma, A.; Zaman, M.F.; Ayazi, F. A Sub-0.2 degrees/hr bias drift micromechanical silicon gyroscope with automatic CMOS mode-matching. IEEE J. Solid-St. Circ. 2009, 44, 1593-1608.

4. Sharma, A.; Zaman, M.F.; Zucher, M.; Ayazi, F. A 0.1 Degrees/Hr Bias Drift Electronically Matched Tuning Fork Microgyroscope. In Proceedings of IEEE 21st International Conference on Micro Electro Mechanical Systems (MEMS 2008), Tucson, AZ, USA, 13-17 January 2008; pp. 6-9.

5. Choi, B.D.; Park, S.; Ko, H.; Paik, S.J.; Park, Y.; Lim, G.; Lee, A.; Lee, S.C.; Carr, W.; Setiadi, D.; et al. The First Sub-Deg/Hr Bias Stability, Silicon-Microfabricated Gyroscope. In Proceedings of IEEE 13th International Conference on Solid-State Sensors, Actuators and Microsystems, Seoul, Korea, 5-9 June 2005; pp. 180-183.

6. Acar, C.; Schofield, A.R.; Trusov, A.A.; Costlow, L.E.; Shkel, A.M. Environmentally robust MEMS vibratory gyroscopes for automotive applications. IEEE Sens. J. 2009, 9, 1895-1906.

7. Saleem, M.M.; Bazaz, S.A. Design and robustness analysis of structurally decoupled 3-DoF MEMS gyroscope in the presence of worst-case process tolerances. Microsyst. Technol. 2011, 17, 1381-1391.

8. Acar, C.; Shkel, A.M. MEMS gyroscopes with structurally decoupled 2-DOF drive and sense mode oscillators. Nanotechnology 2003, 1, 428-431.

9. Acar, C.; Shkel, A.M. Inherently robust micromachined gyroscopes with 2-DOF sense-mode oscillator. J. Microelectromech. Syst. 2006, 15, 380-387.

10. Kang, K.L.; Xie, J.; Tsai, M.L.J. A new robust four degree-of-freedom gyroscope design. Nems/Mems. Technol. Devices 2011, 254, 199-202.

11. Acar, C.; Shkel, A.M. Non-resonant surface-micromachined z-axis gyroscopes utilizing torsional out-of-plane detection. Proc. IEEE Sens. 2004, 1-3, 665-668.

12. Trusov, A.A.; Schofield, A.R.; Shkel, A.M. Study of Substrate Energy Dissipation Mechanism in In-Phase and Anti-Phase Micromachined Vibratory Gyroscopes. In Proceedings of 2008 IEEE Sensors, Lecce, Italy, 26-29 October 2008; pp. 168-171.

13. Cho, C.H. Characterization of Young's modulus of silicon versus temperature using a "beam deflection" method with a four-point bending fixture. Curr. Appl. Phys. 2009, 9, 538-545.

(C) 2013 by the authors; licensee MDPI, Basel, Switzerland. This article is an open access article distributed under the terms and conditions of the Creative Commons Attribution license (http://creativecommons.org/licenses/by/3.0/). 